\title{
Dynamics of harvested-predator-prey model: role of alternative resources
}

\author{
Banshidhar Sahoo $^{1} \cdot$ Barun $_{\text {Das }^{2}} \cdot$ Sukumar Samanta $^{2}$
}

Received: 4 July 2016/Accepted: 11 July 2016/Published online: 25 July 2016

(C) Springer International Publishing Switzerland 2016

\begin{abstract}
In this paper, A predator-prey model with square root functional response for herd behaviour of prey incorporating predator harvesting is proposed and analysed. The predator population is provided with alternative resource. The proposed model is demonstrated in respect of theoretical as well as numerical results. The Pontryagin's maximum principle is used to characterized the optimal harvesting strategy. Bifurcation study with the variation of harvesting effort and alternative resource are done in respect of numerical simulation. Simulation results show that suitable alternative resource has the capability to prevent predator extinction risk at higher harvesting level. The proposed model and obtaining results are usable in the field of conservation of biology.
\end{abstract}

Keywords Predator-prey - Square root response function . Alternative resource $\cdot$ Harvesting $\cdot$ Extinction $\cdot$ Bifurcation

Banshidhar Sahoo

banshivu@gmail.com

Barun Das

bdasskbu@gmail.com

Sukumar Samanta

sukumarvu@yahoo.com

1 Department of Applied Mathematics, University of Calcutta, Kolkata, West Bengal, India

2 Department of Applied Mathematics, S.K.B. University, Purulia, West Bengal, India

\section{Introduction}

The study of predator-prey system with different ecological phenomena becomes a great popularity in ecological science. In ecological system, each of the population systems take various strategy viz. refuging, grouping, etc. for searching of food sources and for defensive purposes. So, the construction of mathematical models depend on various ecological factors and parameters. Huge number of predator-prey systems were investigated and studied theoretically as well as numerically incorporating various ecological phenomena. Mainly mathematical models of predator-prey systems depend on the interaction of prey and predator population. Functional relationship between predator and prey population are the central themes in mathematical ecology. Most of the models were used simple common type of Holling functional responses (cf. Tang et al. 2014; Lu and Zhang 2010; Tang et al. 2014; Zhang et al. 2012, 2014; Jana et al. 2015; Moussaoui and Bouguima 2014; Gakkhar and Gupta 2016). Recently, Sahoo and Poria proposed predator-prey models with general Holling type of interactions (cf. Sahoo and Poria 2014). They established that oscillatory coexistence and stable state of a system were dramatically changed depending on general Holling parameter values. Therefore, a suitable and proper choice of functional response is required to make a model more realistic. Ajraldi et al. (2011) considered predator-prey systems in which interaction terms use the square root of the prey population rather than simply the prey population. Such predator-prey model is considered in which the prey exhibits herd behaviour, so that the predator interacts with the prey along the outer corridor of the herd of prey. Actualy in real situation, a class of prey population exhibits herd behaviour so that the capturing rate of prey by predator is different 
than normal situation. For an instants the capturing rate of zooplankton by a fish in a ocean is greater than the capturing rate of phytoplankton by fish. In this case phytoplankton behaves herd behaviour then zooplankton. The use of the square root properly accounts for the assumption that the interactions occur along the boundary of the population. As a mathematical consequence of the herd behaviour of prey, we consider in this paper a predatorprey model with square root functional response of prey population. Therefore, the predator-prey model with square root functional responses of a logistic prey $(x)$, and predator $(y)$ is of the form (Braza 2012):

$\frac{d x}{d t}=x(1-x)-\frac{\sqrt{x} y}{1+a \sqrt{x}}$,

where " $t$ " is time. The constant " $c$ " is the consumption rate of predator. The parameter " $s$ " represents the death rate of predator and " $a$ " is the half saturation constant. In order to preserve the biological meaning of the model, the parameters are assumed to be strictly positive. The proposed model may be entirely fitting for herbivorous on a large savanna and their large predators.

Harvesting has a strong impact on the dynamics of predator-prey system. In recent years the growing demand for more resources/foods has reported in over exploitation of several biological resources. Therefore there is a need for a sustainable development strategy in various spheres of human activity to protect ecosystems. In particular bioeconomic modeling is concerned with scientific management of the exploitation of renewable resources like fisheries and forestry (cf. Makinde 2007). Hence harvesting of the ecosystems has been of interest to both economists and ecologists for some time now. Dynamics of predator-prey models with harvesting were studied extensively by several researchers (cf. Sahoo and Poria 2013,2014; Kar and Ghosh 2012; Sahoo 2012). The above studies motivate us to focus on the predator-prey model with predator harvesting. We now incorporate the constant harvesting effort " $e$ " on predator population in the model (1) and we obtain

$$
\begin{aligned}
& \frac{d x}{d t}=x(1-x)-\frac{\sqrt{x} y}{1+a \sqrt{x}}, \\
& \frac{d y}{d t}=\frac{c \sqrt{x} y}{1+a \sqrt{x}}-s y-e y .
\end{aligned}
$$

In the absence of harvesting, predator population can be free from extinction risk; however, harvesting can lead to the incorporation of a positive extinction probability. If a population is subjected to a positive extinction rate then harvesting can drive the population density to a dangerously low level at which extinction becomes sure no matter how the harvester affects the population afterwards. To prevent the extinction risk of predator population, the extra alternative resource/alternative food is required. One relevant work regarding alternative food source is done by Spencer and Collie (1996) establishing a model of preypredator fish with alternative prey in presence of harvesting. Recently, many researchers (cf. Sahoo and Poria 2014; Sahoo 2012; Kar and Chattopadhyay 2010; Moitri et al. 2015) investigated harvested predator-prey system with alternative prey. We now introduce some alternative resource(A) to predator population in to the model (2) and the model (2) is modified into the form

$$
\begin{aligned}
& \frac{d x}{d t}=x(1-x)-\frac{\sqrt{x} y}{1+a \sqrt{x}}, \\
& \frac{d y}{d t}=c y\left(\frac{A \sqrt{x}}{1+a \sqrt{x}}+\epsilon(1-A)\right)-s y-e y,
\end{aligned}
$$

where " $A$ " is a time independent constant parameter and its origin is the alternative resource. The parameter " $\epsilon$ " characterizes as the consumption rate of alternative resource. If $A=1$, the predator depends only on the prey species and thus it is clear that the system (2) is a special case of system (3). Notice that if $A=0$, the predator populations grow without any interaction and the growth rate of the predators are determined by alternative prey. In such case, the predation pressure is completely removed and predator populations evolve in presence of alternative resource only. But such decoupled system is out of our interest. A predator which alternates between two sources of food can be represented within $0<A<1$ and $0<\epsilon<1$. We analyse the system (3) by considering $a=0$ as well as $a \neq 0$ to observe complete dynamics of the model. Therefore, incorporating the above fact, the model (3) with $a=0$ becomes

$$
\begin{aligned}
& \frac{d x}{d t}=x(1-x)-\sqrt{x} y, \\
& \frac{d y}{d t}=c y(A \sqrt{x}+\epsilon(1-A))-s y-e y .
\end{aligned}
$$

The systems (3) and (4) have to be analysed with initial conditions $x(0)>0, y(0)>0$.

In this paper, we first consider predator-prey models in presence of alternative resource to predator subjected to predator harvesting. We investigate the effects of harvesting as well as the effects of supplying alternative resource into the models. Our main objective is to survive predator population from extinction in supplying alternative resource. Actually, our target is to control the predator population in presence of alternative resource and harvesting. The section-wise split of this paper is as follows: In Sect. 2, the positivity, boundedness, stability of equilibria and monotonic behaviours are studied theoretically. Bionomic equilibrium point is constructed in Sect. 3. The optimal control strategy is studied in Sect. 4. Section 5 illustrates some of the key findings through numerical simulations. We draw a conclusion in Sect. 6. 


\section{Theoretical studies}

\section{Theoretical dynamics of the system (3)}

In this subsection, we now analyse the full system (3) analytically.

\section{Positive invariance}

System (3) may be written in the matrix form as $\dot{\bar{X}}=G(\bar{X})$ with $\bar{X}(0)=\bar{X}_{0} \in \mathbb{R}_{+}^{2}$, where $\bar{X}=(x, y)^{T} \in \mathbb{R}_{+}^{2}$ and $G(\bar{X})$ is given by

$$
G=G(\bar{X})=\left(\begin{array}{c}
x(1-x)-\frac{\sqrt{x} y}{1+a \sqrt{x}} \\
c y\left(\frac{A \sqrt{x}}{1+a \sqrt{x}}+\epsilon(1-A)\right)-s y-e y
\end{array}\right),
$$

where $G: C_{+} \rightarrow \mathbb{R}^{2}$ and $G \in C^{\infty}\left(\mathbb{R}^{2}\right)$.

It is easy to show that whenever $\bar{X}(0) \in \mathbb{R}_{+}^{2}$ such that $X_{i}=0$ then $\left.G_{i}(\bar{X})\right|_{X_{i}=0} \geq 0$ (for $\mathrm{i}=1,2$ ). Thus any solution of $G=G(\bar{X})$ with $\bar{X}_{0} \in \mathbb{R}_{+}^{2}$, say $\bar{X}(t)=\bar{X}\left(t, \bar{X}_{0}\right)$, is such that $\bar{X}(t) \in \mathbb{R}_{+}^{2}$ for all $t>0$ (cf. Nagumo 1994).

\section{Boundedness}

Theorem 2.1 All solutions of the system (3) which start in $\mathbb{R}_{+}^{2}$ are uniformly bounded if $s+e>\epsilon c(1-A)$ satisfies.

Proof Let $(x(t), y(t))$ be any solution of the system (3) with positive initial conditions.

Let us consider that

$$
\begin{gathered}
v=x+\frac{1}{A c} y, \\
\text { i.e., } \frac{d v}{d t}=\frac{d x}{d t}+\frac{1}{A c} \frac{d y}{d t} .
\end{gathered}
$$

Using equations of (3), we have

$$
\begin{aligned}
& \frac{d v}{d t}=x(1-x)-\frac{\sqrt{x} y}{1+a \sqrt{x}}+\frac{1}{A c} c y \\
& \quad\left(\frac{A \sqrt{x}}{1+a \sqrt{x}}+\epsilon(1-A)\right)-\frac{s+e}{A c} y . \\
& \text { i.e., } \frac{d v}{d t}=-(1-x)^{2}-x+1+\frac{y}{A c} \\
& (c \epsilon(1-A)-s-e) . \\
& \text { i.e., } \frac{d v}{d t} \leq-\left(x+\frac{y}{A c}(s+e-c \epsilon(1-A))\right)+1 . \\
& \text { Thus, } \frac{d v}{d t} \leq-L v+1, \text { where } L=\min \{1, s+e-\epsilon c(1-A)\}, \\
& \text { provided } s+e>\epsilon c(1-A) \text {. Hence, } \frac{d v}{d t}+L v \leq 1 .
\end{aligned}
$$

Applying the theory of differential inequality Birkhoff and Rota (1982) we obtain $0<v<\frac{1-e^{-L t}}{L}+v(x(0)$, $y(0)) e^{-L t}$.

For $t \rightarrow \infty$, we have $0<v<\frac{1}{L}$. Hence all the solutions of (3) that initiate in $\mathbb{R}_{+}^{2}$ are confined in the region $T=\left\{(x, y) \in \mathbb{R}_{+}^{2}: v=\frac{1}{L}+\xi\right.$, for any $\left.\xi>0\right\}$.

Note: The condition $s+e>\epsilon c(1-A)$ implies that $1-\frac{s+e}{c \epsilon}<A<1$. Therefore, for uniformly bounded solutions of the system (3), depends on alternative food depends on harvesting effort $(e)$.

Existence and local stability criteria of equilibrium points

System (3) possesses following equilibrium states:

(a) The trivial equilibrium state is $E_{T} \equiv(0,0)$. An eigenvalue associated with the Jacobian matrix at $E_{0}$ is 1 , positive, for which $E_{T}$ is an unstable equilibrium point.

(b) The axial equilibrium state is $E_{A} \equiv(1,0)$. The Jacobian matrix at the equilibrium point $E_{A}$ is

$$
J\left(E_{A}\right)=\left(\begin{array}{cc}
-1 & \frac{-1}{1+a} \\
0 & c\left(\frac{A}{1+a}+\epsilon(1-A)\right)-s-e
\end{array}\right) .
$$

The eigen values of $J\left(E_{A}\right)$ are -1 and $c\left(\frac{A}{1+a}+\epsilon(1-A)\right)-s-e$. The axial equilibrium point $E_{A}$ is stable if $s+e>\frac{A c}{1+a}+c \epsilon(1-A)-s$, otherwise $E_{A}(1,0)$ is unstable.

(c) The interior equilibrium state is $E^{*} \equiv\left(x^{*}, y^{*}\right)$, where $x^{*}=\left(\frac{s+e-c \epsilon(1-A)}{A c-((s+e)-c \epsilon(1-A)) a}\right)^{2}, \quad y^{*}=\sqrt{x^{*}}\left(1-x^{*}\right)(1+$ $\left.a \sqrt{x^{*}}\right)$. The Jacobian matrix at $E^{*}$ is given by

$$
J\left(E^{*}\right)=\left(\begin{array}{ll}
\gamma_{11} & \gamma_{12} \\
\gamma_{21} & \gamma_{22}
\end{array}\right)
$$

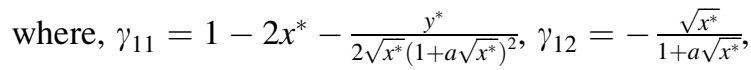
$\gamma_{21}=\frac{A c y^{*}}{2 \sqrt{x^{*}}\left(1+a \sqrt{x^{*}}\right)^{2}}$,

$\gamma_{22}=c\left(A \frac{\sqrt{x^{*}}}{1+a \sqrt{x^{*}}}+\epsilon(1-A)\right)-s-e=0 . \quad$ The characteristic equation of the Jacobian matrix at $E^{*}$ is given by $\lambda^{2}+\Theta_{1} \lambda+\Theta_{2}=0$,

where, $\Theta_{1}=-\gamma_{11}, \Theta_{2}=-\gamma_{12} \gamma_{21}$.

It is clear that $\gamma_{12}<0$, and $\gamma_{21}>0$. Hence the system (3) is stable if $\Theta_{1}>0$, and $\Theta_{1}^{2}-4 \Theta_{1} \Theta_{2}<0$. Thus $E^{*} \equiv$ $\left(x^{*}, y^{*}\right)$ will be stable if $\Theta_{1}>0$ i.e., if $2 x^{*}>\left(1-x^{*}\right)\left(1+\frac{a \sqrt{x^{*}}}{1+a \sqrt{x^{*}}}\right)$. 


\section{Monotonic dynamics}

Proposition 2.1 Equilibrium level of prey biomass of the system (3) decreases monotonically with the increase of alternative resource $A$ if $A c \epsilon<1$ and increases with the increase of harvesting effort $e$.

Proof Now, $x^{*}=\frac{1}{A^{2}}\left(\frac{s+e}{c}-\epsilon(1-A)\right)^{2}$. Differentiating $x^{*}$ with respect to $A$ we get

$\frac{d x^{*}}{d A}=2 \frac{(s+e-c \epsilon(1-A))\left(A c^{2} \epsilon-c\right)}{(A c-(s+e) a+c \epsilon(1-A) a)^{3}}<0$, provided if, $A c \epsilon<1$.

Again Differentiating $x^{*}$ with respect to $e$ we get

$\frac{d x^{*}}{d e}=2 A c\left(\frac{s+e-c \epsilon(1-A)}{(A c-(s+e) a+c \epsilon(1-A) a)^{3}}\right)>0$, since $x^{*}$ is positive.

Hence the proof is completed.

Proposition 2.2 Equilibrium level of predator biomass of the system (3) is an increasing function of alternative resource $A$, but is an decreasing function of $e$ if $a<\frac{3 x^{*}-1}{2 \sqrt{x^{*}}\left(1-2 x^{*}\right)}$.

Proof We have, $y^{*}=\sqrt{x^{*}}\left(1-x^{*}\right)\left(1+a \sqrt{x^{*}}\right)$ Differentiating $y^{*}$ with respect to $A$ we get

$$
\begin{aligned}
& \frac{d y^{*}}{d A}=\left(\frac{\left(1+a \sqrt{x^{*}}\right)\left(1-3 x^{*}\right)}{2 \sqrt{x^{*}}}+\frac{a}{2}\left(1-x^{*}\right)\right) \\
& \frac{d x^{*}}{d A}>0, \text { provided, } a<\frac{3 x^{*}-1}{2 \sqrt{x^{*}}\left(1-2 x^{*}\right)} .
\end{aligned}
$$

Again, differentiating $y^{*}$ with respect to $e$ we have

$$
\begin{aligned}
& \frac{d y^{*}}{d e}=\left(\frac{\left(1+a \sqrt{x^{*}}\right)\left(1-3 x^{*}\right)}{2 \sqrt{x^{*}}}+\frac{a}{2}\left(1-x^{*}\right)\right) \\
& \frac{d x^{*}}{d e}<0, \text { provided, } a<\frac{3 x^{*}-1}{2 \sqrt{x^{*}}\left(1-2 x^{*}\right)} .
\end{aligned}
$$

Hence the proof is completed.

Therefore, from the above Propositions, it is clear that systems biomass of the system (3) depends on supply of alternative resource $A$ and harvesting effort $e$.

\section{Extinction criterion for predator}

Lemma 2.1 If $e>c\left(\frac{A \sqrt{x(r)}}{1+a \sqrt{x(r)}}+\epsilon(1-A)\right)$, then $\lim _{t \rightarrow \infty} y(t)=0$.
Proof The second equation of the system (3) is given by $\frac{d y}{d t}=\left(c\left(\frac{A \sqrt{x(r)}}{1+a \sqrt{x(r)}}+\epsilon(1-A)\right)-s-e\right) y$. Therefore, $\frac{d y}{y} \leq\left(c\left(\frac{A \sqrt{x(r)}}{1+a \sqrt{x(r)}}+\epsilon(1-A)\right)-e\right) d t$.

That is, $y(t) \leq y\left(t_{0}\right) \exp$

$\left(\int_{t_{0}}^{t}-\left(e-c\left(\frac{A \sqrt{x(r)}}{1+a \sqrt{x(r)}}+\epsilon(1-A)\right)\right) d r\right)$.

Thus, $\quad \lim _{t \rightarrow \infty} y(t)=0, \quad$ provided $\quad e>c$ $\left(\frac{A \sqrt{x(r)}}{1+a \sqrt{x(r)}}+\epsilon(1-A)\right)$.

\section{Theoretical studies of the system (4)}

First we study the system (4) theoretically to obtain the existence and stable conditions. The functions of the right hand side of the system (4) are continuous and have continuous partial derivatives on the state space $\mathbb{R}_{+}^{2}=\{(x(t), y(t)): x(t) \geq 0, y(t) \geq 0\}$. Therefore, they are Lipschitzian on $\mathbb{R}_{+}^{2}$ and hence the solution of the system (4) with positive initial conditions exist and unique. Moreover, following Cao et al.( 2012), it is easy to show that the state space $\mathbb{R}_{+}^{2}$ is an invariant domain of the system (4).

In the following Section, positivity and boundedness of solution of the system (4) are to be established. The boundedness may be interpreted as natural restrictions to unlimited growth as a consequence of limited resources (cf. Gakkhar and Singh 2012).

\section{Positive invariance}

System (4) can be written in the matrix form as $\dot{\bar{X}}=F(\bar{X})$ with $\bar{X}(0)=\bar{X}_{0} \in \mathbb{R}_{+}^{2}$, where $\bar{X}=(x, y)^{T} \in \mathbb{R}_{+}^{2}$ and $F(\bar{X})$ is given by

$$
F=F(\bar{X})=\left(\begin{array}{c}
x(1-x)-\sqrt{x} y \\
c y(A \sqrt{x}+\epsilon(1-A))-s y-e y
\end{array}\right),
$$

where $F: C_{+} \rightarrow \mathbb{R}^{2}$ and $F \in C^{\infty}\left(\mathbb{R}^{2}\right)$.

It can be shown that whenever $\bar{X}(0) \in \mathbb{R}_{+}^{2}$ such that $X_{i}=0$ then $\left.F_{i}(\bar{X})\right|_{X_{i}=0} \geq 0$ (for $\mathrm{i}=1,2$ ). Now any solution of $F=F(\bar{X})$ with $\bar{X}_{0} \in \mathbb{R}_{+}^{2}$, say $\bar{X}(t)=\bar{X}\left(t, \bar{X}_{0}\right)$, is such that $\bar{X}(t) \in \mathbb{R}_{+}^{2}$ for all $t>0$ (cf. Nagumo 1994). 


\section{Boundedness}

Theorem 2.2 All solutions of the system (4) which start in $\mathbb{R}_{+}^{2}$ are uniformly bounded if $s+e>\epsilon c(1-A)$ holds.

Proof Let $(x(t), y(t))$ be any solution of the system (4) with positive initial conditions.

Let us assume that $\quad w=x+\frac{1}{A c} y$,

i.e., $\frac{d w}{d t}=\frac{d x}{d t}+\frac{1}{A c} \frac{d y}{d t}$.

Using equations of (4), we have

$$
\begin{aligned}
& \frac{d w}{d t}=x(1-x)-\sqrt{x} y+\sqrt{x} y-\frac{(s+e)}{A c} y+\epsilon \frac{(1-A)}{A} y \\
& \text { i.e., } \frac{d w}{d t} \leq-\theta(x+y)-(x-1)^{2}+1
\end{aligned}
$$

where $\theta=\min \{1, s+e-\epsilon c(1-A)\}$, provided $s+e>$ $\epsilon c(1-A)$.

Therefore, $\frac{d w}{d t}+\theta w \leq 1$.

Applying the theory of differential inequality (Birkhoff and Rota 1982) we obtain $0<w<\frac{1-e^{-\theta t}}{\theta}+w(x(0), y(0)) e^{-\theta t}$.

For $t \rightarrow \infty$, we have $0<w<\frac{1}{\theta}$.

Hence all the solutions of the system (4) that initiate in $\mathbb{R}_{+}^{2}$ are confined in the region

$S=\left\{(x, y) \in \mathbb{R}_{+}^{2}: w=\frac{1}{\theta}+\eta\right.$, for any $\left.\eta>0\right\}$. This proves the theorem.

Note: The condition $s+e>\epsilon c(1-A)$ implies that $1-\frac{s+e}{c \epsilon}<A<1$. Therefore, for uniformly bounded solutions of the system (4), supply of alternative food and harvesting effort $(e)$ depend on each other.

Existence and local stability criteria of equilibrium points

System (4) possesses following three equilibrium states:

(a) The trivial equilibrium state is $E_{0} \equiv(0,0)$. An eigenvalue associated with the Jacobian matrix at $E_{0}$ is 1 , positive, for which $E_{0}$ is an unstable equilibrium point.

(b) The axial equilibrium state is $E_{1} \equiv(1,0)$. The Jacobian matrix at the equilibrium point $E_{1}$ is

$J\left(E_{1}\right)=\left(\begin{array}{cc}-1 & -1 \\ 0 & c(A+\epsilon(1-A))-s-e\end{array}\right)$.

The eigenvalues of $J\left(E_{1}\right)$ are -1 and $c(A+\epsilon(1-A))-s-e$. The axial equilibrium point $E_{1}$ is stable if $e>A c+c \epsilon(1-A)-s$. If the condition is violated, then $E_{1}$ is a saddle point.

(c) The interior equilibrium state is $E_{2} \equiv\left(x^{*}, y^{*}\right)$, where $x^{*}=\frac{1}{A^{2}}\left(\frac{s+e}{c}-\epsilon(1-A)\right)^{2}, y^{*}=\frac{1}{c^{3} A^{3}}[(s+e)-\epsilon(1-$
$\left.A)] \cdot\left[A^{2}-(s+e)-c \epsilon(1-A)\right\}^{2}\right]$. The equilibrium state $E_{2} \quad$ exists if $c \epsilon(1-A)<s+e<A c+$ $c \epsilon(1-A)$. The Jacobian matrix at $E_{2}$ is given by

$J\left(E_{2}\right)=\left(\begin{array}{ll}A_{11} & A_{12} \\ A_{21} & A_{22}\end{array}\right)$,

where, $\quad A_{11}=1-2 x^{*}-\frac{y^{*}}{2 \sqrt{x^{*}}}, \quad A_{12}=-\sqrt{x^{*}}$, $A_{21}=\frac{A c y^{*}}{2 \sqrt{x^{*}}}, \quad A_{22}=c\left[A \sqrt{x^{*}}+\epsilon(1-A)\right]-s-e=0$.

The characteristic equation of the Jacobian matrix at $E_{2}$ is $\lambda^{2}+\Omega_{1} \lambda+\Omega_{2}=0, \quad$ where, $\Omega_{1}=-A_{11}$, $\Omega_{2}=-A_{12} A_{21}$.

It is obvious that $A_{12}<0$, and $A_{21}>0$. Hence the system (4) is stable if $\Omega_{1}>0$, and $\Omega_{1}^{2}-4 \Omega_{1} \Omega_{2}<0$. Thus $E_{2}$ will be stable if $\Omega_{1}>0$ i.e., $e>\frac{A c}{\sqrt{3}}+\epsilon c(1-A)-s$.

\section{Monotonic dynamics}

Proposition 2.3 Equilibrium level of prey biomass (x) decreases monotonically with the increase of alternative resource $A$ if $s+e>c \epsilon$ and increases with the increase of harvesting effort $e$ if $s+e>c \epsilon(1-A)$.

Proof Now, $x^{*}=\frac{1}{A^{2}}\left(\frac{s+e}{c}-\epsilon(1-A)\right)^{2}$. Differentiating $x^{*}$ with respect to $A$ we get

$\frac{d x^{*}}{d A}=\frac{-2}{A^{3}}\left(\frac{s+e}{c}-\epsilon\right)^{2}-\frac{2}{A^{2}} \epsilon\left(\frac{s+e}{c}-\epsilon\right)<0, \quad$ if $s+e>c \epsilon$.

Differentiating $x^{*}$ with respect to $e$ we have

$\frac{d x^{*}}{d e}=\frac{2}{A^{2} c^{2}}(s+e-c \epsilon(1-A))>0, \quad$ if $s+e>c \epsilon(1-A)$.

Hence the proof is completed.

Proposition 2.4 Equilibrium level of predator biomass (y) of the system (4) is an increasing function of with respect to alternative resource $A$ if $A c<\sqrt{3}$ and $s+e>c \epsilon$, but is an decreasing function of $e$ if $A c<\sqrt{3}$ and $s+e>c \epsilon(1-A)$.

Proof We have, $y^{*}=\sqrt{x^{*}}\left(1-x^{*}\right)\left(1+a \sqrt{x^{*}}\right)$ Differentiating $y^{*}$ with respect to $A$ we get

$\frac{d y^{*}}{d A}=\left(1-3 x^{*}\right) \frac{d x^{*}}{d A}$

since $\frac{d x^{*}}{d A}<0$. Therefore, $\frac{d y^{*}}{d A}>0$, if $s+e>c \epsilon$ and $A c<\sqrt{3}$.

Again, differentiating $y^{*}$ with respect to $e$ we have

$\frac{d y^{*}}{d e}=\left(1-3 x^{*}\right) \frac{d x^{*}}{d e}$

since $\frac{d x^{*}}{d e}>0$. Therefore, $\frac{d y^{*}}{d e}<0$, if $s+e>c \epsilon(1-A)$ and $A c<\sqrt{3}$.

Hence the proof is completed. 
Therefore, from the above Propositions, it is clear that prey and predator biomass of the system (4) depends on supply of alternative resource $A$ and harvesting effort $e$.

\section{Extinction criterion for predator}

Lemma 2.2 If $e>c\{A \sqrt{x(r)}+\epsilon(1-A)\}$, then $\lim _{t \rightarrow \infty}$ $y(t)=0$.

Proof The second equation of the system (4) is given by $\frac{d y}{d t}=(c\{A \sqrt{x(r)}+\epsilon(1-A)\}-s-e) y$,

Therefore, $\frac{d y}{y} \leq(c\{A \sqrt{x(r)}+\epsilon(1-A)\}-e) d t$.

i.e., $y(t) \leq y\left(t_{0}\right) \exp$

$\left(\int_{t_{0}}^{t}-((s+e)-c\{A \sqrt{x(r)}+\epsilon(1-A)\}) d r\right)$.

Thus, $\quad \lim _{t \rightarrow \infty} y(t)=0, \quad$ provided $\quad e>c\{A \sqrt{x(r)}+\epsilon$ $(1-A)\}$.

\section{Bionomic equilibrium}

The term bionomic equilibrium is an amalgamation of biological equilibrium and economic equilibrium. The biological equilibrium of the system (3) satisfy the equations

$\frac{d x}{d t}=\frac{d y}{d t}=0$.

The economic equilibrium is said to be achieved when $T R$ (total revenue obtained by selling the harvested predator $y$ ) equals to $T C$ (the total cost for the effort devoted to harvesting). At first, we consider the term $e$ to be the nondimensional measure of the harvesting effort, $p$ is the constant price per unit biomass, $h$ is the constant cost of harvesting effort and $\omega$ is the economic constant. Therefore the economic rent (net revenue) at any time is given by

$\pi(y, e)=T R-T C=(p-\omega e y) e y-h e$.

For convenience, we take $p$ and $h$ to be constant. So, the economic equilibrium can be obtained from the Eq. (5) and using the equation of zero profit line

$$
\pi(y, e)=0 \text {. i.e., }(p-\omega e y) e y-h e=0,
$$

Therefore, $\omega e y^{2}-p y+h=0$.

Thus, using the Eqs. (5) and (7) one can obtain the feasible economic equilibrium $(\hat{x}, \hat{y})$. The optimal economic rent is calculated in the next section.

\section{Optimal control strategy}

The main aim of this section is on the profit-making aspect of the model (3). It is a thorough study of the optimal harvesting strtegy to minimize the profit earned by harvesting biomass considering quadratic costs and conservation of predator. It is assumed that price is a function which decreases with the increase of harvested biomass. Thus, to maximize the total discounted net revenues from the model (3), the optimal control problem can be formulated (cf. Chakraborty et al. 2012) as

$J(e)=\int_{t_{0}}^{t_{f}} e^{-\delta t}[(p-\omega e y) e y-h e] d t$,

where $\delta$ is the instantaneous annual discount rate. The problem (8) can be solved by using Pontryagins maximum principle subject to model (3) and control constraints $0 \leq e \leq e_{\max }$. The convexity of the objective function with respect to $e$, the linearity of the differential equations in the control and the compactness of the range values of the state variables can be combined to give the existence of the optimal control.

Assume that $e_{\delta}$ is an optimal control harvesting with corresponding states $x_{\delta}, y_{\delta}$. We consider $A_{\delta}\left(x_{\delta}, y_{\delta}\right)$ as optimal equilibrium point. Now derive optimal control $e_{\delta}$ such that

$J\left(e_{\delta}\right)=\max \{J(e): e \in U\}$, where $U$ is the control set defined by $U=\left\{e:\left[t_{0}, t_{f}\right] \rightarrow\left[0, e_{\max }\right] \mid e\right.$ is Lebesgue measurable\}.

The Hamiltonian of this optimal control problem is of the form

$$
\begin{aligned}
H= & (p-\omega e y) e y-h e+\lambda_{1}\left\{x(1-x)-\frac{\sqrt{x} y}{1+a \sqrt{x}}\right\} \\
& +\lambda_{2}\left\{c y\left(\frac{A \sqrt{x}}{1+a \sqrt{x}}+\epsilon(1-A)\right)-s y-e y\right\},
\end{aligned}
$$

where $\lambda_{1}, \lambda_{2}$ are adjoint variables. Here the initial transversality conditions give $\lambda_{i}\left(t_{f}\right)=0, i=1,2$.

Now, it is possible to find the characterization of the optimal control $e_{\delta}$. On the set $t: 0<e_{\delta}(t)<e_{\max }$, we have

$\frac{\partial H}{\partial e}=p y-2 \omega y^{2} e-h-\lambda_{2} y$.

At $A_{\delta}\left(x_{\delta}, y_{\delta}\right)$ and $e=e_{\delta}(t)$ we have

$\frac{\partial H}{\partial e}=p y_{\delta}-2 \omega y_{\delta}^{2} e_{\delta}-h-\lambda_{2} y_{\delta}=0$.

Therefore, $e_{\delta}=\frac{p y_{\delta}-h-\lambda_{2} y_{\delta}}{2 \omega y_{\delta}^{2}}$.

Now, the adjoint equations at the point $A_{\delta}\left(x_{\delta}, y_{\delta}\right)$ are 
(a)

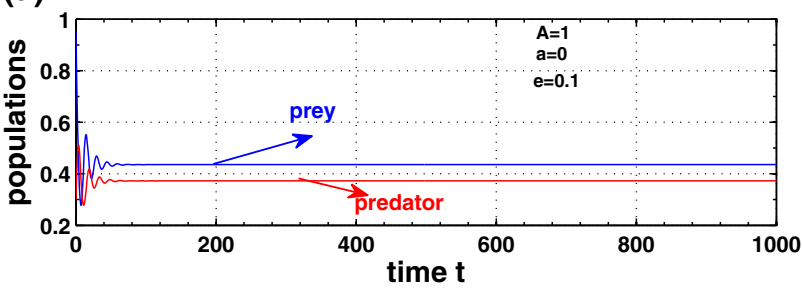

(c)

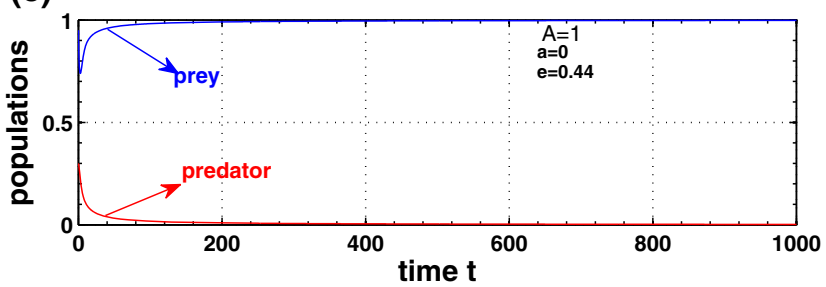

(b)

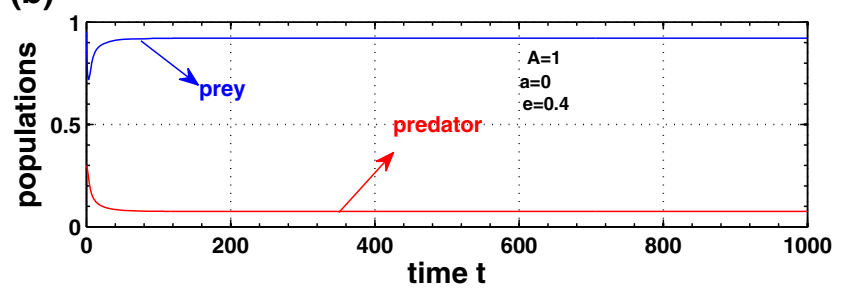

(d)

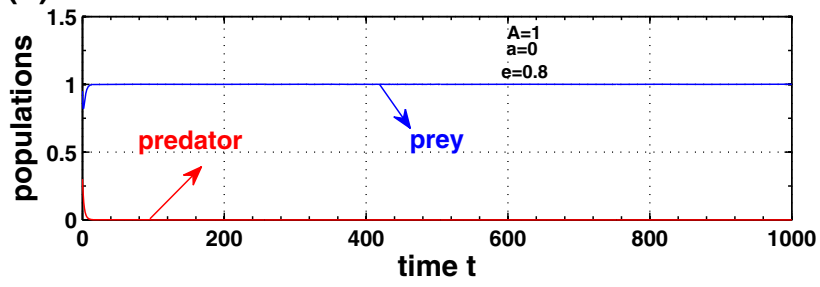

Fig. 1 Dynamics of the system (4) taking $a=0, c=1, s=0.56, \epsilon=0.95$ and $A=1$ with various harvesting effort $e=0.1$ for (a), $e=0.4$ for (b),$e=0.44$ for (c) and $e=0.8$ for (d). Figure depicts that predator population has extinction risk for higher harvesting effort

$$
\begin{aligned}
\frac{d \lambda_{1}}{d t}= & \delta \lambda_{1}-\left.\frac{\partial H}{\partial x}\right|_{A_{\delta}}, \\
= & \delta \lambda_{1}-\left[\lambda_{1}\left(1-2 x_{\delta}-\frac{y_{\delta}}{2 \sqrt{x_{\delta}}\left(1+a \sqrt{x_{\delta}}\right)^{2}}\right)\right. \\
& \left.+\lambda_{2}\left(\frac{A c y_{\delta}}{2 \sqrt{x_{\delta}}\left(1+a \sqrt{x_{\delta}}\right)^{2}}\right)\right], \\
\frac{d \lambda_{2}}{d t}= & \delta \lambda_{2}-\left.\frac{\partial H}{\partial y}\right|_{A_{\delta}}, \\
= & \delta \lambda_{2}-\left[\lambda_{2}\left(\left(\frac{A c \sqrt{x_{\delta}}}{\left(1+a \sqrt{x_{\delta}}\right)}\right)+\epsilon(1-A)-\left(s+e_{\delta}\right)\right)\right. \\
& \left.-\lambda_{1} \frac{\sqrt{x_{\delta}}}{1+a \sqrt{x_{\delta}}}\right]-\left(p e_{\delta}-2 \omega e_{\delta}^{2} y_{\delta}\right) .
\end{aligned}
$$

The two Eqs. (11) and (12) are first order simultaneous differential equations and it is easy to get the analytical solution of the equations with the help of initial conditions $\lambda_{i}\left(t_{f}\right)=0, i=1,2$. Using the value of $\lambda_{2}$ and Eqs. (5) and (9), we can get the feasible optimum harvesting equilibrium $\left(x_{\delta}, y_{\delta}\right)$. Therefore the optimum economic rent or net revenue at any time is obtained using the value of $y_{\delta}$ from the Eq. (6). In this regard, it is important to note that we have formulated the optimal control problem considering harvesting effort as control parameter. We summarize the above study by the following theorem.

Theorem 4 There exists an optimal control $e_{\delta}$ and corresponding solutions of the system (3), the equilibrium $\left(x_{\delta}\right.$, $\left.y_{\delta}\right)$ maximizes $J(e)$ over $U$. Furthermore, there exists adjoint functions $\lambda_{1}, \lambda_{2}$ and $\lambda_{2}$ satisfying Eqs. (11) and (12) with transversality conditions $\lambda_{i}\left(t_{f}\right)=0, i=1,2$. Moreover, the optimal control is given by $e_{\delta}=\frac{p y_{\delta}-h-\lambda_{2} y_{\delta}}{2 \omega y_{\delta}^{2}}$.

\section{Numerical studies}

The numerical simulations of the system (3) (for $a \neq 0$ ) and the system (4) (for $a=0$ ), are demonstrated with a set of fixed parameter values $c=1, s=0.56$ and $\epsilon=0.95$, most of which are taken from Braza (2012). The remaining two parameters A $(0<A<1)$ and "e" are varied throughout the simulations.

\section{Numerical results for $a=0$}

We first simulate the dynamics of the predator population of the system (4) without alternative resource (i.e., for $A=1$ ) for different harvesting effort (e) presenting in Fig. 1. Figure 1a displays that prey and predator population have stable dynamics for $e=0.1$. When "e" is increasing, predator density decreases (Fig. 1b). We also observe that the predator population will extinct in the system after $e \geq 0.44$ (Fig. 1c, d). So we now study to observe the effects of alternative resource (A) when $e \geq 0.44$. The effects of alternative resource $(A)$ is represented in Fig. 2 fixing harvesting effort at $e=0.44$. From Fig. 2, it is evident that the predator population has also extinction risk in presence of alternative resource. Figure 2 shows that alternative resource has no capability to prevent the extinction or extinction risk with this harvesting.

\section{Numerical results for $a \neq 0$}

We now study the dynamics of the system (3) for different harvesting effort $e$ in absence of alternative resource (i.e., $A=1$ ) taking $a=0.2$ and it is presented in 
(a)

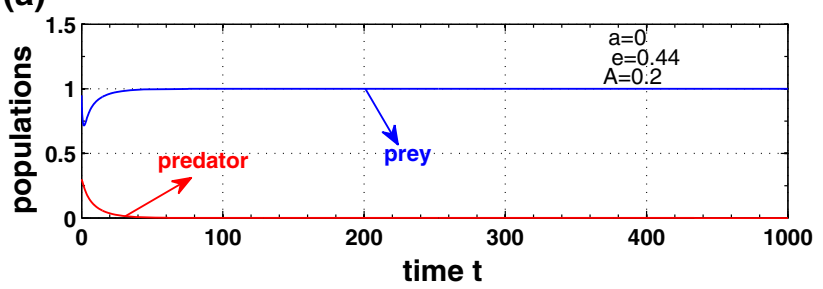

(c)

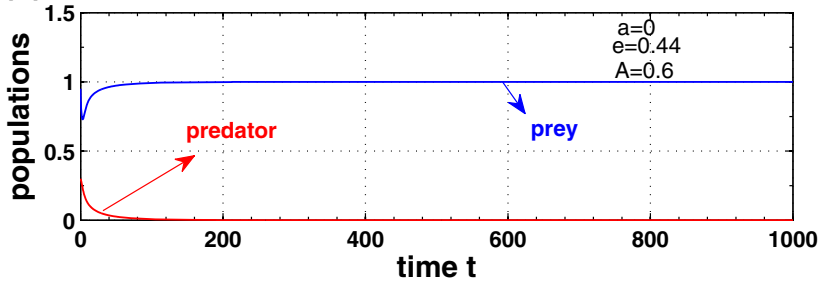

Fig. 2 Dynamics of the system (4) taking $a=0, c=1, \epsilon=0.95$, $s=0.56$ and $e=0.44$ in presence of alternative resource $A=0.2$ for (a), $A=0.4$ for (b),$A=0.6$ for (c) and $A=0.9$ for (d). (b)

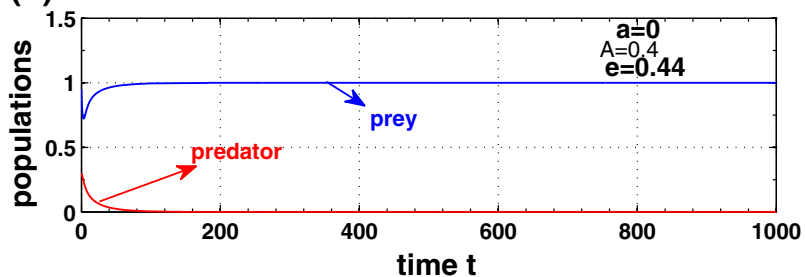

(d)

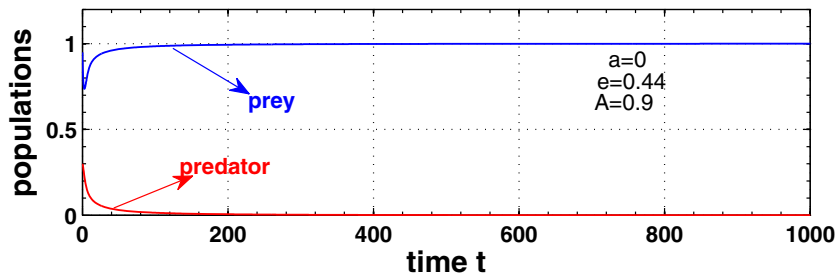

Figure displays that predator extinction risk deos not reduce for higher harvesting effort and presence of alternative resource

(b)
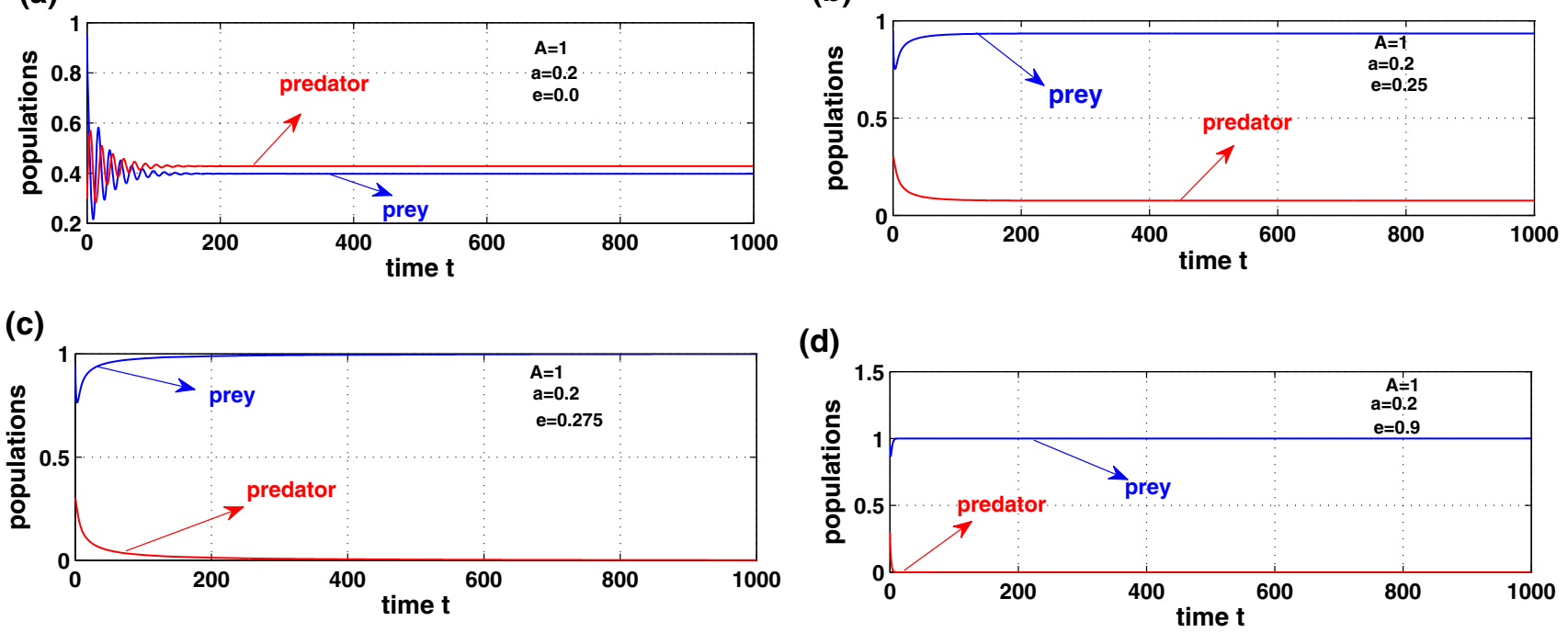

(d)

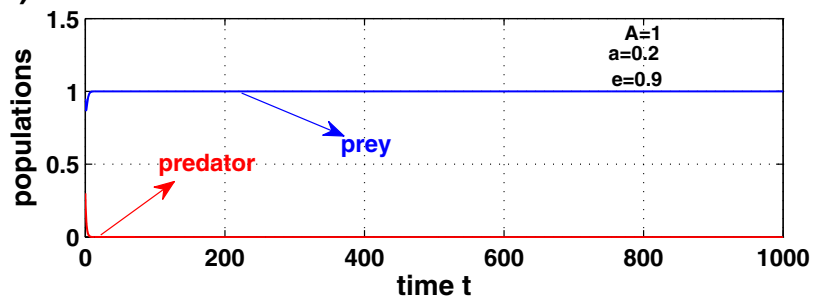

Fig. 3 Dynamics of the system (3) taking $a=0.2, c=1, \epsilon=0.95, s=0.56$ and $A=1$ with various harvesting effort $e=0$ for (a), $e=0.25$ for (b),$e=0.275$ for (c) and $e=0.9$ for (d). It is evident that predator population has extinction risk for higher harvesting rate

Fig. 3. Figure 3(a) depicts that prey and predator population have stable dynamics for $e=0$. The density of predator population decreases for increase rate of harvesting $(e=0.25)$ (Fig. 3b). It is evident that predator populations will have extinction when $e \geq 0.275$ (Fig. 3c, d). Now the bifurcation diagram of the system (3) with respect to harvesting effort " $\mathrm{e}$ " is presented in Fig. 4 in absence of alternative resource $(\mathrm{A}=1)$. From Fig. 4, we observe that predator population will extinct for $e \geq 0.275$, whereas prey population exists in their highest density level. The dynamics of the system (3) in presence of alternative resource is presented in Fig. 5 fixing harvesting effort at $e=0.3$. Figure 5a-c depicts that the predator populations survive in the system in presence of alternative resource (A) . Predator population will have extinction in presence of alternative resource (Fig. 4d). It is clear from Fig. 5 that the predator population will survive for $0.27 \leq A<0.79$. Therefore, predator population of the system (3) will exist for suitable supply of alternative resource (A). The arbitrary supply of alternative resource may have opposite effect (Fig. 5d). The role of alternative resource (A) is presented in Fig. 6 through 
Fig. 4 Bifurcation of the system (3) with respect to harvesting effort e without alternative resource (i.e., for A $=1$ ). Figure displays that predator population will extinct for $e \geq 0.275$

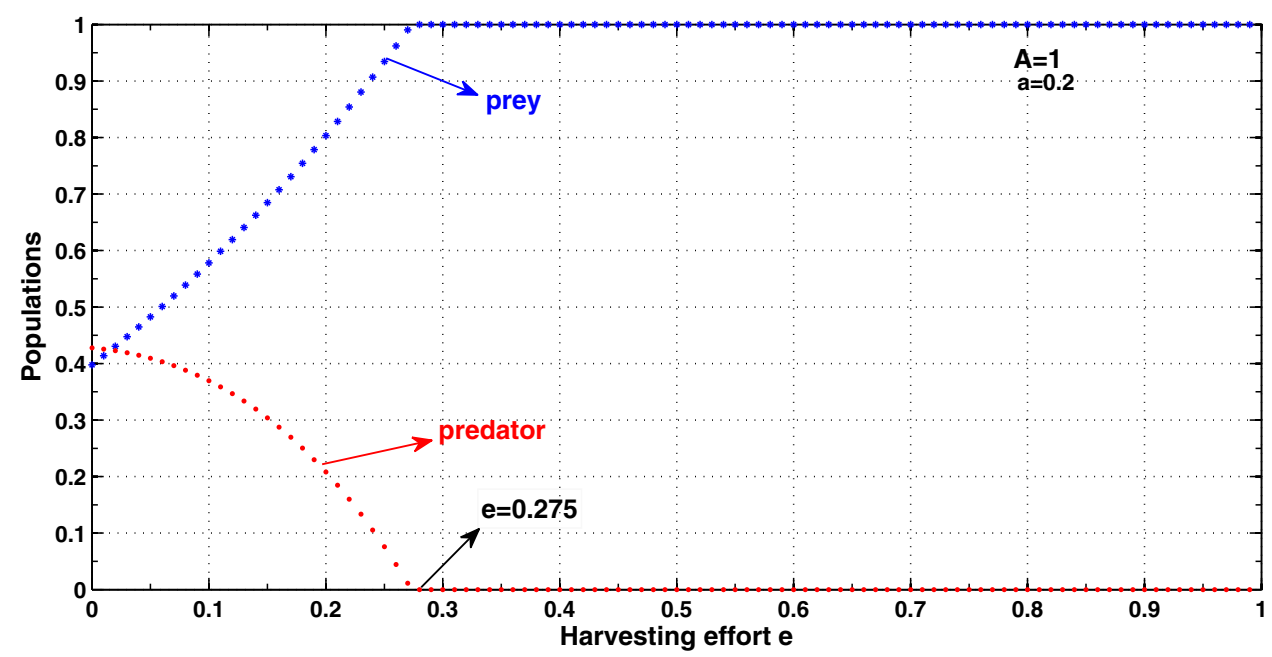

(a)

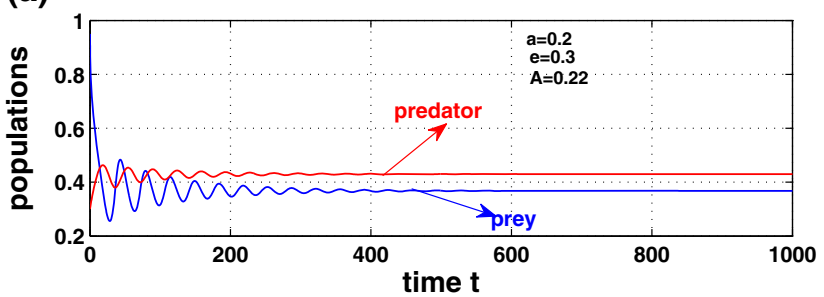

(c)

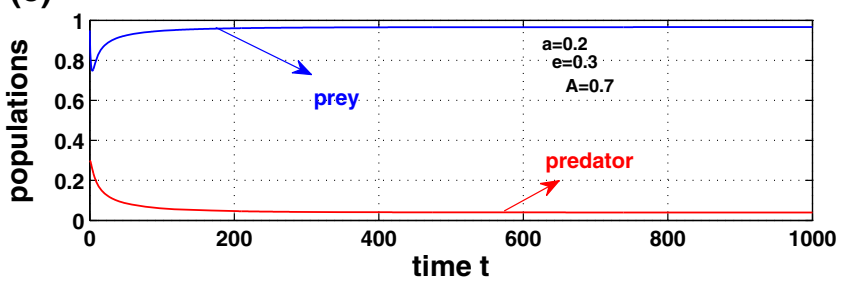

Fig. 5 Dynamics of the system (3) taking $a=0.2, c=1, \epsilon=0.95$, $s=0.56$ and $e=0.3$ in presence of alternative resource $A=0.22$ for (a), $A=0.5$ for (b) , $A=0.7$ for (c) and $A=0.79$ for (d).

bifurcation analysis for fixed harvesting effort $e=0.3$. From Fig. 6, one can observe that predator survives for $0<A<0.79$ and have extinction for $A \geq 0.79$. The functional relation of minimum level of alternative resource (A) with maximum harvesting effort (e) is presented in Fig. 7. Figure 7 displays that one can choose the suitable alternative resource for survival of predator population when harvesting effort becomes high.

\section{Dynamics for seasonal harvesting}

We now focus on the dynamics of the system (3) when harvesting is time dependent. In reality, parameter values are not fixed, should be variable. In different season, harvesting may be changed with time. So, we now consider that harvesting depends on seasonality. We (b)

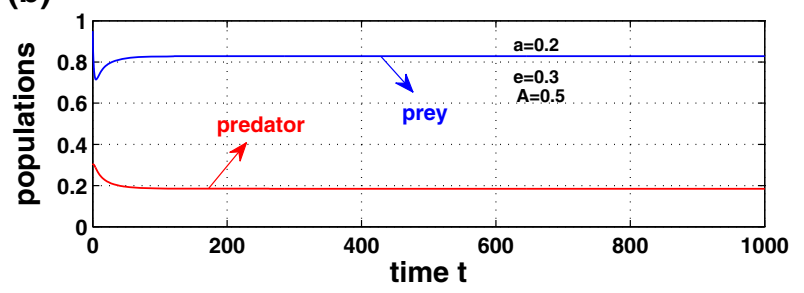

(d)

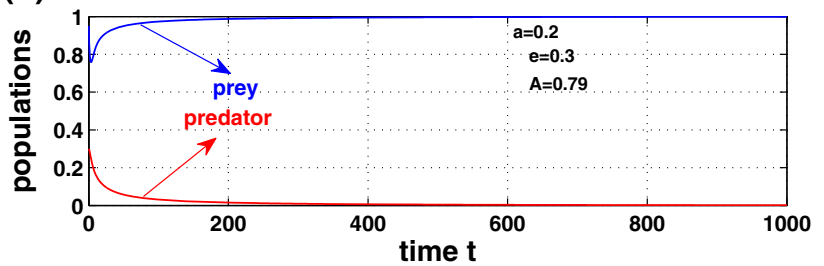

Figure displays that predator population will survive in the system for higher harvesting rate with suitable supply of alternative resource

replace harvesting effort $e$ by $e\left(1+\delta_{1} \cos \phi t\right)$ in the model (3), where " $\delta_{1}$ " represents amplitude of oscillation and " $\phi$ " represents angular frequency of oscillation. We draw a bifurcation diagram of the system (3) with respect to $e$ in absence of alternative resource $(A=1)$ for $s=0.56, c=1, \epsilon=0.95, \delta_{1}=0.1$ and $\phi=0.5$ in Fig. 8. From Fig. 8, we observe that the predator population oscillates within $0 \leq e<0.28$ and have extinction for $e \geq 0.28$. Taking $e=0.3$ as fixed, we plot the dynamics of the system (3) with respect to alternative resource $A$ in figure 9. Figure 9 shows that predator population will survive within $0<A<0.79$ and will extinct for $0.79 \leq A \leq 1$. Therefore, one can conclude that alternative resource has a capability to prevent the extinction probability of predator incorporating time dependent harvesting effort. 
Fig. 6 Bifurcation of the system (3) with respect to alternative resource $\mathrm{A}$ with constant harvesting effort $\mathrm{e}=$ 0.3 . Figure depicts that predator population exists positively for $0<A<0.79$ and may extinct for $A \geq 0.79$

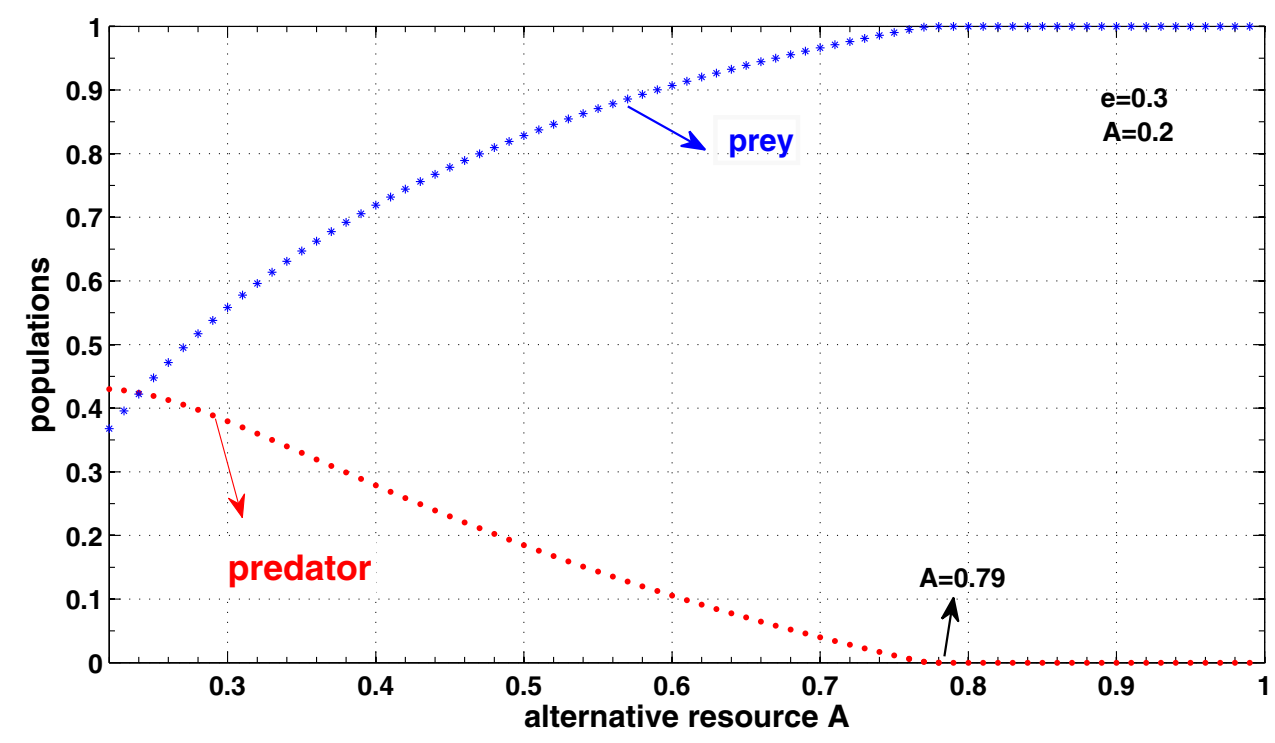

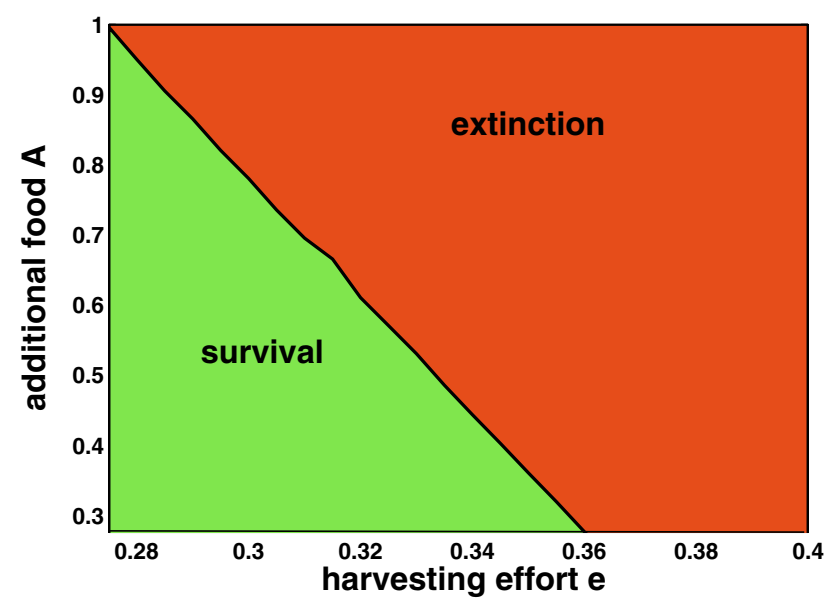

Fig. 7 Dynamical behaviour of the predator population of the system (3) in $e-A$ plane taking $a=0.2, \epsilon=0.95, c=1, s=0.56$. Figure depicts that one can choose the suitable minimum level alternative food for higher harvesting rate

\section{Results for optimal harvesting strategy}

The effects of variation of constant price per unit biomass of catch (p) and alternative resource (A) on optimal control of harvesting effort $e_{\delta}$ in the model (3) is described in the Fig. 10. Figure 10 displays that optimal harvesting effort $\left(e_{\delta}\right)$ decreases with time. But for smaller to higher price rate, the level of optimal harvesting effort $\left(e_{\delta}\right)$ increases (Fig. 10a) when supply of alternative resource is fixed. On the other hand for a fixed price rate, the level of $e_{\delta}$ increases with respect to rapid supply of alternative resource. Therefore, it is clear that maximum level of harvesting, depends on market price value as well as supply of alternative resource.

\section{Conclusion}

We have proposed a predator-prey model with predator harvesting in presence of alternative resource. We have first studied the conditions of preliminary properties like positivity, boundedness, stability of equilibrium points etc. for both models. The bionomic equilibrium of the system is obtained and the optimal control strategy is also studied. The theoretical results are verified through numerical simulations. Bifurcation analysis is presented with the variation of harvesting effort as well as alternative resource. From bifurcation analysis, we observe that predator population will extinct for $e \geq 0.275$ in absence of alternative resource (Fig. 4). On the other hand, Fig. 6 depicts that predator population exists positively for $0<A<0.79$ for fixed harvesting effort $e=0.3$ and will have extinction risk for $A \geq 0.79$. Therefore, a suitable supply of alternative resource can prevent the extinction risk of predator. A dynamical behaviour of the predator population of the system (3) in $e-A$ plane is plotted in Fig. 7 and it shows that one can choose the minimum level of suitable alternative resource for higher harvesting rate. We have also presented the system dynamics with time varying harvesting effort in Figs. 8 and 9. Figures 8 and 9 show that system has similar dynamics in presence of time varying harvesting effort with time independent harvesting effort. Lastly, we have described the result of optimal harvesting effort in Fig. 10 to observe the effects of alternative resource and market price rate.

Thus, we observe that a supply of suitable alternative resource $A$ can reduce the predation pressure on prey as well as remove extinction possibility of predator 
Fig. 8 Bifurcation of the system (3) with respect to harvesting effort e without alternative resource (i.e., for $\mathrm{A}$ $=1$ ) in presence of seasonality. Figure displays that predator population will extinct for $e \geq 0.275$

Fig. 9 Bifurcation of the system (3) with respect to alternative resource (A) for fixed harvesting $(e=0.3)$ in presence of seasonality. Figure shows that predator population will survive in higher harvesting with suitable alternative resource
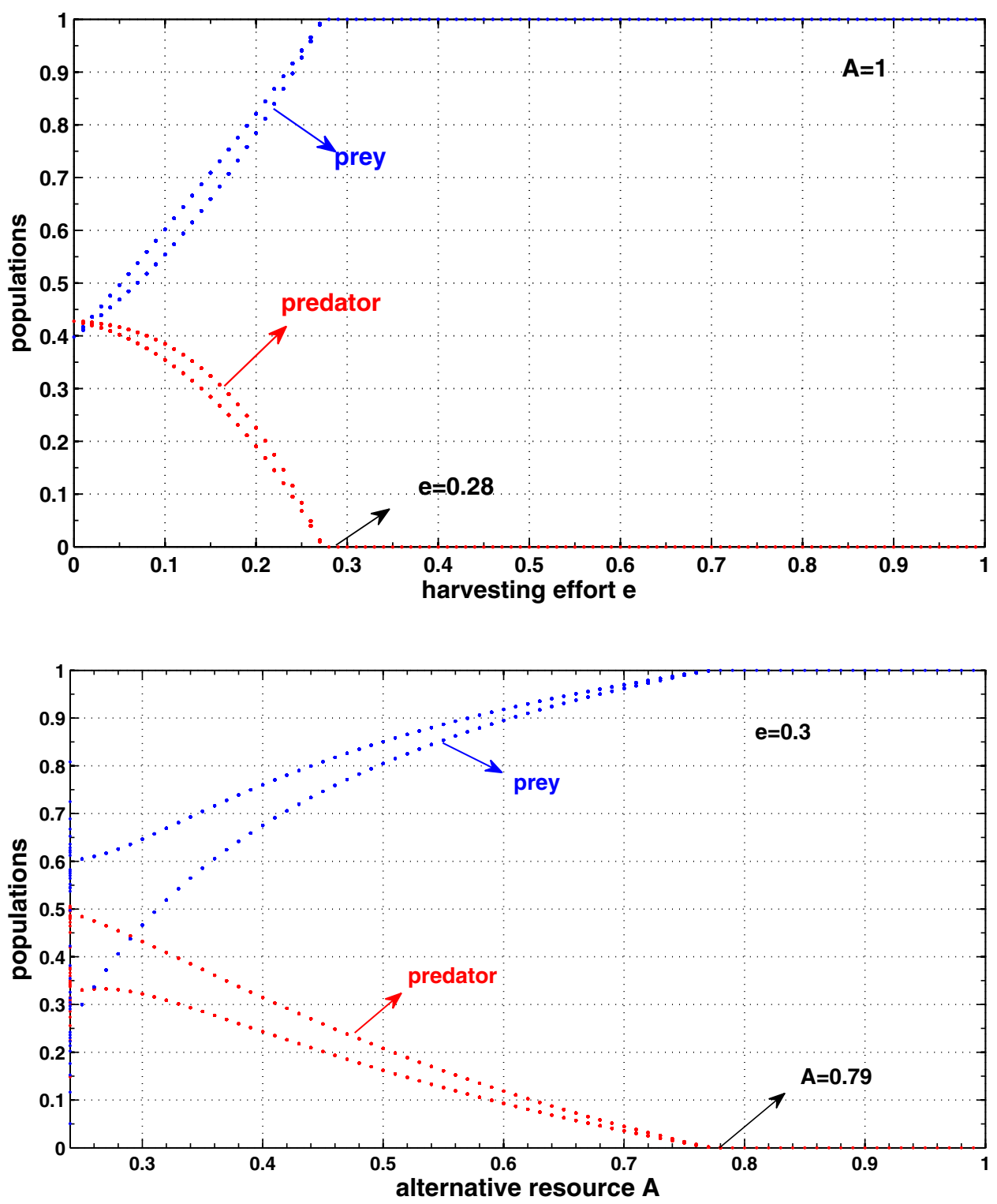

(a)

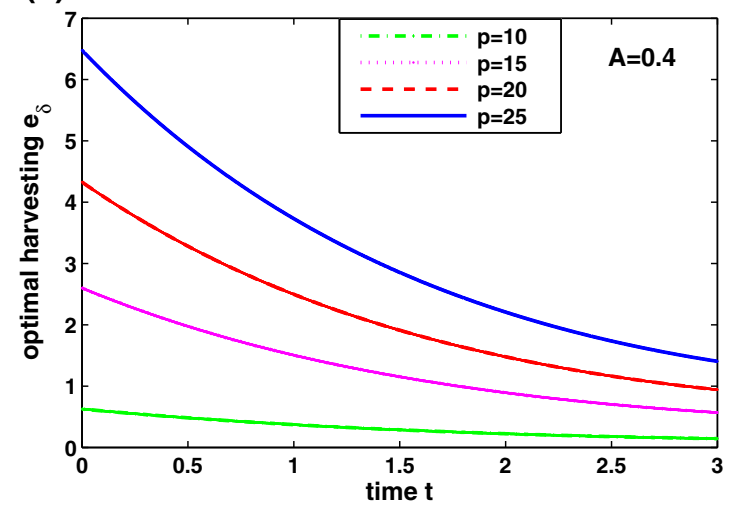

Fig. 10 a Variation of optimal control of harvesting effort $e_{\delta}$ with the increasing time and with the variation of constant price per unit biomass of catch for $a=0.2, \delta=0.4, e=0.3, s=0.56, c=1$, $A=0.4, \omega=10.5$. b Variation of optimal control of harvesting effort (b)

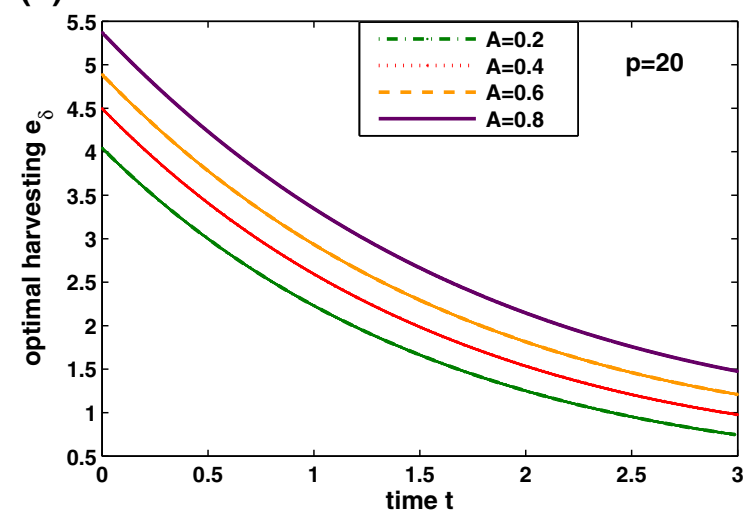

$e_{\delta}$ with the increasing time with the variation of alternative food per unit biomass (A) for $a=0.2, \delta=0.4, e=0.3, s=0.56, c=1$, $p=20, \omega=10.5$ 
population. In this paper, we assume that the supply of alternative resource is not dynamic, but maintained at a specific constant level. This simplification is justified for many arthropod predators, because they can feed on plantprovided alternative resources such as pollen or nectar (cf. Baalen et al. 2011). This model is especially important in such systems in which more energy enters the food web from alternative resource. The results may be applicable in the field of conservation of biological as well as fishery.

\section{References}

Ajraldi V, Pittavino M, Venturino E (2011) Modeling Herd behavior in population systems. Nonlinear Anal RWA 12:2319-2338

Birkhoff G, Rota GC (1982) Ordinary differential equations. Ginn Boston

Braza PA (2012) Predator-prey dynamics with square root functional responses. Nonlinear Anal 13:1837-1843

Chakraborty K, Jana S, Kar TK (2012) Global dynamics and bifurcation in a stage structured prey-predator fishery model with harvesting. Appl Math Comput 218:9271-9290

Feng C, Chen L (1998) Asymptotic behavior of nonautonomous diffusive Lotka-Volterra model. Syst Sci Math Sci 11:107-111

Gakkhar S, Gupta K (2016) A three species dynamical system involving prey-predation, competition and commensalism. Appl Math Comput 273:54-67

Gakkhar S, Singh A (2012) Control of chaos due to additional predator in the Hastings-Powell food chain model. J Math Anal Appl 385(1):423-438

Jana S, Ghorai A, Guria S, Kar TK (2015) Global dynamics of a predator, weaker prey and stronger prey system. Appl Math Comput 250:235-248

Kar TK, Chattopadhyay SK (2010) A focus on long-run sustainability of a harvested prey predator system in the presence of alternative prey. Comptes Rendus Biol 333:841-849

Kar TK, Ghosh B (2012) Sustainability and optimal control of an exploited prey predator system through provision of alternative food to predator. Biosystems 109:220-232

Liu C, Zhang Q, Li J, Yue W (2014) Stability analysis in a delayed prey-predator-resource model with harvest effort and stage structure. Appl Math Comput 238:177-193

Lu C, Zhang L (2010) Permanence and global attractivity of a discrete semi-ratio dependent predator-prey system with Holling II type functional response. J Appl Math Comput 33:125-135
Makinde OD (2007) Solving ratio-dependent predator-prey system with constant effort harvesting using Adomian decomposition method. Appl Math Comput 186:17-22

Moussaoui A, Bouguima SM (2014) Seasonal influences on a preypredator model. J Appl Math Comput 50:1-19

Nagumo M (1994) Uber die Lage der Integralkurven gew onlicher Differentialgleichungen. Proc Phys Math Soc 24:551

Sahoo B, Poria S (2013) Disease control in a food chain model supplying alternative food. Appl Math Model 37:5653-5663

Sahoo B, Poria S (2014) Diseased prey predator model with general Holling type interactions. Appl Math Comput 226:83-100

Sahoo B, Poria S (2014) Oscillatory coexistence of species in a food chain model with general Holling interactions. Diff Equ Dyn Syst 22(3):221-238

Sahoo B, Poria S (2014) The chaos and control of a food chain model supplying additional food to top-predator. Chaos Solitons Fractals 58:52-64

Sahoo B, Poria S (2014) Effects of supplying alternative food in a predator-prey model with harvesting. Appl Math Comput 234:150-166

Sahoo B, Poria S (2014) Effects of allochthonous resources in a three species food chain model with harvesting. Differ Equ Dyn Syst 23:257-279

Sahoo B (2012) Effects of additional foods to predators on nutrientconsumer-predator food chain model. ISRN Biomath

Sahoo B (2012) Global stability of predator-prey system with alternative prey. ISRN Biotech

Sen Moitri, Srinivasu PDN, Malay Banerjee (2015) Global dynamics of an additional food provided predator-prey system with constant harvest in predators. Appl Math Comput 250:193-211

Spencer PD, Collie JS (1996) A simple predator-prey model of exploited marine fish populations incorporating alternative prey. ICES J Mar Sci 53:615-628

Tang G, Tang S, Cheke RA (2014) Global analysis of a Holling type II predator-prey model with a constant prey refuge. Nonlinear Dyn 76:635-647

van Baalen M, Krivan V, van Rijn PCJ, Sabelis MW (2011) Alternative food, switching predators, and the persistence of predator-prey systems. Am Nat 157:512-524

Zhang Z, Yang H, Fu M (2014) Hopf bifurcation in a predator-prey system with Holling type III functional response and time delays. J Appl Math Comput 44:337-356

Zhang Q, Liu C, Zhang X (2012) Bifurcations of a singular predatorprey model with Holling-II functional response. Complex. Anal Control Biol Syst :113-125

Zhang X, Wu Z, ZhouT (2016) Permanence of a predator-prey discrete system with Holling-IV functional response and distributed delays. J Biol dyna 10:1-17 